\title{
Response Styles to Positive Affect and Depression: Concurrent and Prospective Associations in a Community Sample
}

\author{
Sabine Nelis $\cdot$ Emily A. Holmes $\cdot$ Filip Raes
}

Published online: 8 February 2015

(c) Springer Science+Business Media New York 2015

\begin{abstract}
We examined the concurrent and prospective relations between response styles to positive affect and depression in a community sample. Participants $(n=345)$ completed self-report measures of current and past depressive episodes, depressive symptoms, anhedonia, and responses to positive affect (including dampening and positive rumination) at two time points, with a 5-month interval. Higher levels of dampening responses to positive affect were related to higher concurrent levels of depressive symptoms. The tendency to positively ruminate on positive affect was negatively related to concurrent anhedonic symptoms. When controlling for current depressive symptomatology, formerly depressed individuals had a higher tendency to dampen positive affect than never-depressed controls, and did not differ from a currently depressed group. Dampening responses did not predict depressive symptoms prospectively, but lower levels of (self-focused) positive rumination did predict higher levels of future anhedonic symptoms. Results indicate that not only currently but also formerly depressed individuals engage in dysfunctional (dampening) strategies in response to positive affect. It is possible that currently as well as formerly depressed individuals might benefit from
\end{abstract}

S. Nelis $(\varangle) \cdot$ F. Raes

Faculty of Psychology and Educational Sciences,

KU Leuven - University of Leuven, Tiensestraat 102,

Box 3717, 3000 Leuven, Belgium

e-mail: sabine.nelis@ppw.kuleuven.be

E. A. Holmes

MRC Cognition and Brain Sciences Unit, Cambridge, UK

E. A. Holmes

Department for Clinical Neuroscience, Karolinska Institutet,

Stockholm, Sweden interventions that are directed at the remediation of disturbed regulation of positive affect. However, our prospective results make clear that more research is needed to examine the precise conditions under which dampening would be a detrimental (and positive rumination a beneficial) response style in the course of depression.

Keywords Affect regulation - Rumination - Dampening · Positive affect · Depression · Prediction

\section{Introduction}

Depression is a mental disorder characterised by increased negative affect as well as anhedonia, which refers to reduced positive affect or reduced pleasure or interest in daily activities (American Psychiatric Association 2013, p. 160). Interestingly, the way in which people (maladaptively) regulate their negative affect is related to depression. For example, depressive rumination, which is characterised by repetitively thinking about the causes, meanings, and consequences of one's negative affect (Nolen-Hoeksema 1991) is associated with more (severe) depressive symptoms (Aldao et al. 2010; Thomsen 2006), and predicts depression onset (e.g., Nolen-Hoeksema et al. 2008 for a review). However, we know less about response styles to positive affect. There is growing interest in positive affect in depression (see Dunn 2012) and transdiagnostically (for reviews, see Carl et al. 2013; Gilbert 2012; Hechtman et al. 2013).

One response or thinking style to positive affect that has recently attracted research attention is 'dampening'. Dampening refers to "the tendency to respond to positive moods states with mental strategies to reduce the intensity and duration of the positive mood state" (Feldman et al. 
2008, p. 509). For instance, in a context when one feels (initially) happy, dampening might involve thinking that one did not deserve it; that it was just good luck; or thinking about things that did not go well. An alternative response is so-called 'positive rumination', "the tendency to respond to positive affective states with recurrent thoughts about positive self-qualities, positive affective experience, and one's favourable life circumstances" (Feldman et al. 2008, p. 509). The Responses to Positive Affect scale (RPA, Feldman et al. 2008) assesses positive rumination and dampening regulation strategies which both attempt to modify a positive emotion (Feldman et al. 2008). Furthermore, the RPA, based on the results of factor analyses, distinguishes between two forms of positive rumination: one focuses on mood and somatic experiences (i.e., Emotion-Focus; e.g., notice how you feel full of energy), and another focuses on aspects of the self and pursuit of personally relevant goals (i.e., Self-Focus; e.g., I am achieving everything, Feldman et al. 2008).

Higher levels of dampening have been found to predict higher levels of concurrent depressive symptoms in nonclinical student samples (Feldman et al. 2008; Raes et al. 2009; Werner-Seidler et al. 2013), even above and beyond ruminative responses to negative affect (Feldman et al. 2008; Raes et al. 2009). A positive cross-sectional association between dampening and depressive symptoms has also been reported in children (Bijttebier et al. 2012) and in a heterogeneous group of students diagnosed with major depressive disorder, bipolar disorder or no mood disorder (Johnson et al. 2008). Dampening is also weakly associated with lifetime history of depressive symptoms (Eisner et al. 2009). This general correlational pattern is represented in a group approach. For example, dysphoric students have been found to self-report significantly higher levels of dampening than controls (Nelis et al. 2013); as have currently depressed individuals compared to never-depressed controls (Werner-Seidler et al. 2013).

The relationship between positive rumination and depressive symptoms is not as straightforward as the relationship between dampening and depression. Whereas some studies report a negative relation between emotionfocused positive rumination and depressive symptoms (Raes et al. 2009; Werner-Seidler et al. 2013, Study 2), other studies only observed such a negative relation for the self-focused type of positive rumination (e.g., Feldman et al. 2008). And still, some research studies have found no relation at all between the two types of positive rumination and depressive symptoms (e.g., Johnson et al. 2008).

From these cross-sectional studies it is unclear whether dysfunctional regulation of positive affect is a characteristic of the acute phase of depression only, or also occurs in formerly depressed individuals. In addition to this, it unclear what the directional nature of the relation is (also see:
Werner-Seidler et al. 2013): Does dysfunctional regulation of positive affect predict increases in depressive symptoms over time or/and do depressive symptoms predict an increase in the engagement in dysfunctional positive affect regulation?

We are aware of three studies that examined the relation between responses to positive affect and depression history. In two studies, people with a history of depression did not report significantly higher levels of dampening responses to positive affect than never-depressed controls (Johnson et al. 2008; Werner-Seidler et al. 2013, study 3). On the other hand, a third study observed a higher level of dampening in formerly depressed as compared to neverdepressed individuals (Werner-Seidler et al. 2013, study 2) but this was influenced by current levels of depressive symptoms. Positive rumination, across the three studies, was not significantly related to a history of depression. Findings are limited by relatively small sample sizes.

What do the few prospective studies that were conducted so far tell us about the directional nature of the association between positive affect regulation and depression (or depressive symptoms)? Dampening responses to positive affect predicted depressive symptoms after 3 and 5 months in a non-clinical sample, even when controlling for baseline depressive symptoms and ruminative responses to negative affect (Raes et al. 2012). However, this was not found in a remitted depressed group (prediction of depressive symptoms during a 6-month follow-up period; Gilbert et al. 2013) or in children (3-month period, Bijttebier et al. 2012; short interval (4-12 days), Gentzler et al. 2012). In the adult studies, positive rumination was not a protective factor for later depressive symptoms (Gilbert et al. 2013; Raes et al. 2012).

In the present study, we examined the concurrent and prospective relations between depressive symptoms, and responses to positive affect (dampening and positive rumination) in a large community sample. The first aim was to investigate the relation between depressive symptoms and dampening. Given the robust previous findings, we predicted that depressive symptoms would positively relate to dampening. We also predicted that depressive symptoms would be negatively associated with positive rumination. We made no specific predictions with regard to the two subtypes of positive rumination (emotion and self-focused) given earlier mixed findings. Second, responses to positive affect might be particularly important for a specific core characteristic of depression that is related to positive affect, i.e., anhedonia. Preliminary evidence suggests that responses to positive affect relate to anhedonic symptoms (Werner-Seidler et al. 2013). Therefore, we also investigated the relation of response styles with anhedonic symptoms. Third, to investigate whether dampening depends on depression status, we compared a currently 
depressed group, formerly depressed group and never-depressed group. We predicted that dampening would be lowest in a never-depressed group and highest in a currently depressed group. Given previous research (Johnson et al. 2008: Werner-Seidler et al. 2013), we did not expect group differences for positive rumination. Fourth, following first seminal but also inconsistent findings on the prospective value of dampening in predicting depressive symptoms (Bijttebier et al. 2012; Gilbert et al. 2013; Raes et al. 2012), we expected that dampening would predict higher levels of depressive symptoms (and specifically anhedonia) at a 5-month follow-up. This is the first study to prospectively investigate anhedonia and response styles to positive affect. Finally, to explore the direction of the relation between depression (anhedonia) and response styles to positive affect, we examined the converse; namely whether depressive (or anhedonic) symptoms predict an increase in dysfunctional positive affect regulation (i.e., increased dampening and/or decreased positive rumination)? A last aim was to extend the current research to a more heterogeneous community sample (Raes et al. 2012) with a larger sample size (Gilbert et al. 2013).

\section{Methods}

\section{Participants}

Participants were community volunteers recruited in Belgium via e-mail and social media. At baseline, participants who completed all measurements were included. We made an exception for one participant who had a missing value for only the last scale (concentration, see measures). The resulting sample $(n=345)$ comprised 260 women and 85 men, age $18-82$ years $(M=31.2, S D=12.3)$. Seventynine percent had a higher education degree, $3 \%$ had a professional education after secondary school, $17 \%$ completed secondary school, and $1 \%$ completed grammar school. For the follow-up, again, participants who completed all measures were included $(n=293$, $M$ age $=32.0, S D=12.5,218$ women). Ten randomly selected participants received a gift voucher in exchange for participation.

Measures

Major Depression Questionnaire (MDQ; Van der Does et al. 2003)

The MDQ is a self-report questionnaire that assesses the presence of current and past major depressive episodes. It is based on the DSM-IV criteria for past and current major depression (American Psychiatric Association 1994).
Questions are focused on the past month and are repeated for any time in their life. The MDQ at Time 1 assessed the presence of a current episode at Time 1 and of past depressive episodes. The MDQ at follow-up assessed the presence of a depressive episode at follow-up and at any point during the 5-month follow-up period. Williams et al. (2008) reported evidence for convergent validity with diagnoses from the Structured Clinical Interview for DSMIV (First et al. 1996).

\section{Responses to Positive Affect Scale (RPA;}

Feldman et al. 2008)

The RPA is a 17 -item questionnaire assessing responses to positive affect rated from 1 (almost never) to 4 (almost always). Three subscales are: Dampening (e.g., "My streak of luck is going to end soon", 7 items), Self-focused positive rumination (e.g., "I am achieving everything", 4 items), and Emotion-focused positive rumination (e.g., "Think about how happy you feel", 5 items). The Dutch version with 16 items has adequate psychometric properties (Raes et al. 2009). Internal consistency in the current sample was satisfactory, both at Time 1 (Cronbach's alpha $=0.82,0.74,0.84$ for Dampening, Emotion-focus, and Self-focus, respectively; $n=345$ ) and at follow-up $($ Cronbach's alpha $=0.83,0.73,0.83 ; n=293$ ).

\section{Depression Subscale of the Depression Anxiety Stress} Scales, Short Version (DASS-D; Lovibond and Lovibond 1995)

The (short version) depression subscale of the DASS (DASS-D) was administered. It assesses the severity of depressive symptoms for the past week. Seven items are scored from 0 (did not apply to me at all) to 3 (applied to me very much or most of the time). The Dutch version by de Beurs et al. (2001) has good psychometric properties (de Beurs et al. 2001; Nieuwenhuijsen et al. 2003). Cronbach's alpha in our sample was 0.87 at Time $1(n=345)$ and 0.89 at follow-up $(n=293)$.

Short form of the Dutch adaptation of the Mood and Anxiety Symptoms Questionnaire (MASQ-D30; Wardenaar et al. 2010)

The MASQ-D30 is an adaptation of the original MASQ developed by Watson and colleagues (Watson et al. 1995a, b). The MASQ was developed to measure the dimensions of the tripartite model of Clark and Watson (1991). The MASQ-D30 has three subscales: General Distress, Anhedonic Depression, and Anxious Arousal. We assessed the 10-item Anhedonic scale (MASQ-A) which measures the lack of positive affect. Participants indicated on a 5-point 
scale, from not at all to very much, how they felt during the past week (e.g., I felt like I was having a lot of fun, I felt optimistic, I felt like I had a lot to look forward to). The items are positively formulated; therefore, responses were reversed to capture anhedonia. The MASQ-D30 has good psychometric properties (Wardenaar et al. 2010). Cronbach's alpha in the present sample was 0.93 at Time 1 $(n=345)$ and 0.94 at follow-up $(n=293)$.

\section{Concentration}

Participants were asked to what extent they had been able to complete the online tasks with concentration (without being distracted). Ratings ranged from Not at all (1) to Very good. I have completed all questions with concentration (9). We wanted to check whether participants in the three diagnostic groups (based on the MDQ) would differ on how focused they were while completing the questions.

\section{Procedure}

Participants were recruited via e-mail and social media. The message was available on an online recruiting site of the Faculty of Psychology and Educational Sciences (University of Leuven). The researcher also invited family and friends to participate and to distribute the invitation message (snowball sampling). The invitation contained the address of an online survey tool. On the corresponding website, participants gave their informed consent. Next, they completed the MDQ, RPA, DASS-D, MASQ-A, and a short memory task (in that order-the memory task is not reported in the present paper). The study ended with sociodemographic questions on age, gender, and education, and the concentration scale. Five months later, participants received an invitation via e-mail to complete the second part of the study. They completed the same questionnaires and tasks with an adaptation concerning time in the MDQ (see measures). The study was approved by the Ethical Committee of the Faculty of Psychology and Educational Sciences, University of Leuven.

\section{Results}

\section{Sample Characteristics}

Means, standard deviations, and ranges for all measures at baseline and at follow-up are presented in Table 1. At baseline, based on the self-report MDQ, $8 \%$ of the participants was currently depressed, $26 \%$ had been depressed in the past but were not currently depressed (i.e., 'formerly depressed group'), and $66 \%$ were not currently depressed and had never been depressed before either (i.e., 'never-depressed group'). Twenty-eight of the formerly depressed individuals reported one past depressive episode, 22 individuals reported 2 episodes, and 25 individuals reported three or more episodes. As expected, level of depressive symptoms was higher for the currently depressed group than for the other two groups ( $p$ s $<0.001$ ), while the formerly depressed and never-depressed groups did not significantly differ $(p=0.13)$. On the MASQ-A, the currently depressed group reported higher levels of anhedonic symptoms than the two other groups ( $p$ s $<0.001)$. The formerly depressed and never-depressed group did not significantly differ on the MASQ-A, $p=0.10$; see Table 2. At follow-up ( $n=293), 17$ participants $(6 \%)$ met criteria for current depression according to the MDQ, and $23(8 \%)$ of the participants who were not depressed at baseline $(n=273)$ suffered from a major depressive episode at some point during the follow-up period, as assessed with the MDQ.

Correlational Analyses: Associations Between General Depressive Symptoms, Anhedonia, and Responses to Positive Affect

To investigate the relation between depression and responses to positive affect, the three subscales of the RPA were correlated with the DASS-D (Pearson correlations; Table 3). As predicted, DASS-D was positively associated with RPA-Dampening, and, less strongly, negatively associated with the two positive rumination scales (comparison of the correlations with Steiger's $Z$ tests, $p s<0.001)$.

Next, the relation between responses to positive affect and a specific characteristic of depression (i.e., anhedonia) was investigated. As predicted, higher levels of anhedonia were associated with more dampening and less positive rumination. However, the association between RPADampening and the MASQ-A disappeared after controlling for the DASS-D. Importantly, the DASS-D also includes several anhedonia items which makes it a suboptimal control in the correlation with anhedonia. However, the conclusion remained the same when the association between RPA-Dampening and the MASQ-A was partialled out for (only) the non-anhedonia items of the DASS-D. Moreover, the association between RPA-Dampening and the DASS-D remained after controlling for the MASQ-A, suggesting that dampening is in our sample not uniquely associated with the anhedonic symptom part of depression.

Group Comparisons, According to Depression Group, for Responses to Positive Affect

ANOVAs were conducted to investigate differences between the three groups on the self-reported responses to 
Table 1 Descriptive information for baseline and follow-up measures

DASS-D $=$ Depression subscale of the Depression Anxiety Stress Scales, MASQAnhedonia $=$ Anhedonic Depression subscale of the Short form of the Dutch adaptation of the Mood and Anxiety Symptoms Questionnaire (MASQ-D30), RPA $=$ Responses to Positive Affect Scale: Dampening subscale (RPA-Dampening), Emotion-focused positive rumination subscale (RPAEmotionFocus), Self-focused positive rumination subscale (RPA-SelfFocus)

\begin{tabular}{lrrrr}
\hline & Mean & SD & Min & Max \\
\hline Baseline $(n=345)$ & & & & \\
DASS-D & 3.61 & 3.73 & 0 & 21 \\
MASQ-Anhedonia & 28.50 & 8.17 & 11 & 50 \\
RPA-Dampening & 12.69 & 3.93 & 7 & 26 \\
RPA-EmotionFocus & 13.23 & 2.74 & 5 & 20 \\
RPA-SelfFocus & 8.97 & 2.79 & 4 & 16 \\
Follow-up $(n=293)$ & & & & \\
DASS-D & 3.91 & 3.96 & 0 & 21 \\
MASQ-Anhedonia & 29.49 & 8.24 & 10 & 50 \\
RPA-Dampening & 12.83 & 4.08 & 7 & 26 \\
RPA-EmotionFocus & 12.87 & 2.63 & 7 & 19 \\
RPA-SelfFocus & 8.90 & 2.75 & 4 & 16 \\
Baseline measures for participants included at follow-up $(n=293)$ & & & & \\
DASS-D & 3.57 & 3.67 & 0 & 21 \\
MASQ-Anhedonia & 28.50 & 8.16 & 11 & 50 \\
RPA-Dampening & 12.55 & 3.82 & 7 & 26 \\
RPA-EmotionFocus & 13.17 & 2.77 & 5 & 20 \\
RPA-SelfFocus & 8.93 & 2.78 & 4 & 16 \\
\hline
\end{tabular}

Table 2 Descriptive information at baseline per group

\begin{tabular}{|c|c|c|c|c|c|c|c|c|c|}
\hline & \multicolumn{3}{|c|}{$\begin{array}{l}\text { Currently depressed } \\
n=27\end{array}$} & \multicolumn{3}{|c|}{$\begin{array}{l}\text { Formerly depressed } \\
n=89\end{array}$} & \multicolumn{3}{|c|}{$\begin{array}{l}\text { Never-depressed } \\
n=229\end{array}$} \\
\hline & Mean & $\mathrm{SD}$ & MinMax & Mean & SD & MinMax & Mean & $\mathrm{SD}$ & MinMax \\
\hline Age & 28.3 & 11.6 & & 32.2 & 12.4 & & 31.1 & 12.3 & \\
\hline Gender ( $\%$ female $)$ & 78 & & & 81 & & & 73 & & \\
\hline DASS-D & $9.19^{\mathrm{a}}$ & 5.26 & $1-21$ & $3.57^{\mathrm{b}}$ & 3.17 & $0-14$ & $2.97^{\mathrm{b}}$ & 3.15 & $0-14$ \\
\hline MASQ-A & $38.33^{\mathrm{a}}$ & 8.50 & $14-50$ & $28.80^{\mathrm{b}}$ & 7.81 & $12-49$ & $27.22^{\mathrm{b}}$ & 7.48 & $11-50$ \\
\hline RPA-D & $16.70^{\mathrm{a}}$ & 4.67 & $8-26$ & $13.39^{\mathrm{a}}$ & 3.98 & $7-24$ & $11.95^{\mathrm{b}}$ & 3.47 & $7-23$ \\
\hline RPA-SF & $7.70^{\mathrm{a}}$ & 2.95 & $4-15$ & $8.99^{\mathrm{a}}$ & 3.17 & $4-16$ & $9.11^{\mathrm{a}}$ & 2.58 & $4-15$ \\
\hline RPA-EF & $12.11^{\mathrm{a}}$ & 2.71 & $6-18$ & $13.21^{\mathrm{a}}$ & 2.95 & $5-20$ & $13.36^{\mathrm{a}}$ & 2.63 & $8-19$ \\
\hline
\end{tabular}

DASS-D = Depression subscale of the Depression Anxiety Stress Scales, MASQ-A = Anhedonic Depression subscale of the Short form of the Dutch adaptation of the Mood and Anxiety Symptoms Questionnaire (MASQ-D30), RPA = Responses to Positive Affect Scale: Dampening subscale (D), Emotion-focused positive rumination subscale (EF), Self-focused positive rumination subscale (SF)

a,b Means in the same row with a different subscript differ significantly $(p<0.05)$. For the RPA, subscripts represent the analyses after controlling for DASS-D

positive affect (RPA scales), namely the never-depressed, formerly depressed, and currently depressed groups (as classified by the self-report MDQ). Descriptives are presented in Table 2. Groups significantly differed on RPADampening, $F(2,342)=22.03, p<0.001, \eta_{p}^{2}=0.11$. The formerly depressed group scored significantly higher on RPA-Dampening than the never-depressed group, $F(1$, $316)=10.24, p=0.002, \eta_{p}^{2}=0.03, d=0.40$, and significantly lower than the currently depressed group, $F(1$, $114)=13.21, p<0.001, \eta_{p}^{2}=0.10, d=0.80$. Accordingly, the currently depressed had a higher mean RPA-
Dampening score than the never-depressed group, Welch's $F(1,29.48)=26.36, p<0.001, d=1.32$.

To investigate whether these group differences represented a difference in depression history or rather a difference due to current depressive symptomatology, we controlled for DASS-D. The main effect of condition on RPA-Dampening remained significant, $F(2,341)=6.64$, $p=0.001, \eta_{p}^{2}=0.04$. Importantly, the formerly depressed group continued to score significantly higher than the never-depressed group on RPA-Dampening, $F(1$, $315)=8.03, p=0.005, \eta_{p}^{2}=0.03$. This suggests that the 
Table 3 Correlations between depressive symptoms (DASS-D), dampening, self-focused positive rumination, emotion-focused positive rumination, and anhedonia at baseline

\begin{tabular}{lllll}
\hline & DASS-D & RPA-D & RPA-SF & RPA-EF \\
\hline DASS-D & - & & & \\
RPA-D & $0.45^{* * *}(0.34 * * *)$ & - & & \\
RPA-SF & $-0.16^{* *}$ & -0.05 & - & - \\
RPA-EF & $-0.16^{* *}$ & 0.004 & $0.64 * * *$ & $-0.44^{* * * * *}$ \\
MASQ-A & $0.70^{* * *}$ & $0.31^{* * *}(-0.01)$ & $-0.44^{* * *}$ & - \\
\hline
\end{tabular}

$n=345$. Pearson correlations are presented. Correlations between parentheses represent partial correlations (controlling for DASS-D or MASQ-A). DASS-D = Depression subscale of the Depression Anxiety Stress Scales, RPA = Responses to Positive Affect Scale: Dampening subscale (D), Emotion-focused positive rumination subscale (EF), Self-focused positive rumination subscale (SF); MASQ-A = Anhedonic Depression subscale of the Short form of the Dutch adaptation of the Mood and Anxiety Symptoms Questionnaire (MASQ-D30)

** $p<0.01 ; * * * p<0.001$

initial difference was not explained by depressive symptomatology. However, the formerly depressed group no longer differed significantly from the currently depressed group in terms of RPA-Dampening scores, $F(1$, 113) $=0.23, p=0.63, \eta_{p}^{2}=0.002$. This indicates that the initial difference on RPA-Dampening between formerly and currently depressed individuals was due to the group difference on the current symptoms (DASS-D). The currently depressed group continued to have a higher level of RPADampening than the never-depressed group when controlling for DASS-D scores, $F(1,253)=9.51, p=0.002$, $\eta_{p}^{2}=0.04$. With regard to the two positive rumination scales of the RPA, groups no longer differed on any of the two scales (RPA-SelfFocus and RPA-EmotionFocus) when controlling for current symptoms (DASS-D). ${ }^{1}$

Groups did not differ in how much they concentrated during the online measures and tasks, $p=0.76$. Therefore, group differences are unlikely to be attributed to differences in level of distraction/concentration during assessment.

\section{Prospective Analyses}

Participants who completed the follow-up assessment $(n=293)$ did not significantly differ from non completers $(n=52)$ in terms of depressive symptoms, dampening, positive rumination, and MASQ-Anhedonia at baseline, $p s>0.11$, for descriptives of the follow-up sample, see Table 1. To examine the predictive value of responses to positive affect for depressive symptoms at 5 months follow-up, a hierarchical regression analysis was performed (see Table 4). Depressive symptoms (DASS-D) at followup was the dependent variable. In Step 1, depressive symptoms at Time 1 was included alongside history of a

\footnotetext{
${ }^{1}$ In the comment section of the MDQ, one person-who is included in the formerly depressed group-indicated bipolar disorder. Conclusions of the group comparisons remained the same after exclusion of this person.
}

major depressive episode [no previous episode coded as 0 ( $n=198)$, current and/or previous episode(s) coded as 1 $(n=95)$ ]. We controlled for a history of depression, given that a history of depression is a known predictor of the course of depression (e.g., Eberhard-Gran et al. 2002; Karsten et al. 2011). In Step 2, the three subscales of the RPA were entered. Both models were significant ( $p s<0.001)$. In Step 1, higher levels of baseline depressive symptoms as well as a (positive) history of depression predicted higher levels of depressive symptoms at followup, and explained $29 \%$ of the variance in depressive symptoms. Unexpectedly, in Step 2, responses to positive affect did not add significantly to the explained variance of later depressive symptoms $\left(\Delta R^{2}=0.01, p=0.29\right)$.

To investigate whether dampening predicted anhedonia, the hierarchical regression was repeated with DASS-D replaced by MASQ-Anhedonia (see Table 4). Both models were significant $(p<0.001)$. In Step $1\left(R^{2}=0.26\right)$, a (positive) history of depression predicted higher levels of anhedonic symptoms at follow-up (above baseline anhedonic symptoms). In Step 2, responses to positive affect added significantly to the explained variance of anhedonic symptoms $\left(\Delta R^{2}=0.03, p=0.01\right)$. More specifically, lower levels of self-focused positive rumination significantly predicted higher levels of anhedonic symptoms at follow-up ( $\beta=-0.20)$. Dampening, however, was not a significant predictor. $^{2}$

\footnotetext{
2 Although Self-Focused and Emotion-Focused positive rumination have been identified as two different factors (Raes et al. 2009), Selffocused and Emotion-focused positive rumination were highly correlated in the current sample $(r=0.64)$ and related similarly to the DASS-D and MASQ-A (see Table 3). Treating the scales as one positive rumination scale (Cronbach's alpha $=0.86$ ) revealed no association between positive rumination and depressive symptoms at follow-up, $\beta=0.03$ (after controlling for baseline symptoms, history of depression, and dampening). With regard to the prediction of anhedonia, positive rumination had a negative association with follow-up anhedonic symptoms, $\beta=-0.12, p=0.04$ (after controlling for baseline anhedonia, history of depression and dampening).
} 
Table 4 Hierarchical regression analysis for the prediction of depressive symptoms at follow-up

\begin{tabular}{lrrrrr}
\hline & $B$ & SE $B$ & $\beta$ & \multicolumn{1}{l}{$p$} & $R^{2}$ \\
\hline DV: DASS-D-T2 & & & & & \\
Step 1 & & & & & \\
DASS-D-T1 & $\mathbf{0 . 5 1}$ & $\mathbf{0 . 0 6}$ & $\mathbf{0 . 4 8}$ & $<\mathbf{0 . 0 0 1}$ & \\
Past MDE & $\mathbf{1 . 3 1}$ & $\mathbf{0 . 4 3}$ & $\mathbf{0 . 3 3}$ & $\mathbf{0 . 0 0 3}$ & 0.29 \\
Step 2 & & & & & \\
DASS-D-T1 & $\mathbf{0 . 5 0}$ & $\mathbf{0 . 0 6}$ & $\mathbf{0 . 4 6}$ & $<\mathbf{0 . 0 0 1}$ & \\
Past MDE & $\mathbf{1 . 2 6}$ & $\mathbf{0 . 4 4}$ & $\mathbf{0 . 3 2}$ & $\mathbf{0 . 0 0 4}$ & \\
RPA-EF & 0.15 & 0.09 & 0.11 & 0.11 & \\
RPA-SF & -0.11 & 0.09 & -0.08 & 0.24 & \\
RPA-D & 0.05 & 0.06 & 0.05 & 0.36 & 0.30 \\
DV: MASQ- $A-T 2$ & & & & & \\
Step 1 & & & & & \\
MASQ-A-T1 & $\mathbf{0 . 4 4}$ & $\mathbf{0 . 0 5}$ & $\mathbf{0 . 4 4}$ & $<\mathbf{0 . 0 0 1}$ & \\
Past MDE & $\mathbf{2 . 9 5}$ & $\mathbf{0 . 9 2}$ & $\mathbf{0 . 3 6}$ & $\mathbf{0 . 0 0 1}$ & 0.26 \\
Step 2 & & & & & \\
MASQ-A-T1 & $\mathbf{0 . 3 9}$ & $\mathbf{0 . 0 6}$ & $\mathbf{0 . 3 9}$ & $<\mathbf{0 . 0 0 1}$ & \\
Past MDE & $\mathbf{3 . 1 3}$ & $\mathbf{0 . 9 2}$ & $\mathbf{0 . 3 8}$ & $<\mathbf{0 . 0 0 1}$ & \\
RPA-EF & 0.20 & 0.20 & 0.07 & 0.32 & \\
RPA-SF & $-\mathbf{0 . 6 0}$ & $\mathbf{0 . 2 0}$ & $-\mathbf{0 . 2 0}$ & $\mathbf{0 . 0 0 3}$ & \\
RPA-D & -0.12 & 0.12 & -0.05 & 0.32 & 0.28 \\
\hline
\end{tabular}

$n=293 . \mathrm{DV}=$ Dependent variable. Dependent variable is measured at follow-up (T2). All predictors are measured at baseline (T1). Significant predictors are in bold. $\beta$ 's indicate the regression coefficients when predictors (except Past MDE) and the dependent variable are standardised. DASS-D = Depression subscale of the Depression Anxiety Stress Scales, RPA = Responses to Positive Affect Scale: Dampening subscale (D), Emotion-focused positive rumination subscale (EF), Self-focused positive rumination subscale (SF); MASQ$\mathrm{A}=$ Anhedonic Depression subscale of the Short form of the Dutch adaptation of the Mood and Anxiety Symptoms Questionnaire (MASQ-D30). Past MDE = history of depression: previous/current episode(s) or no previous episode

When history of depression was removed from the analyses, then responses to positive affect did still not add significantly to the explained variance of depressive symptoms (DASS-D), $\Delta R^{2}=0.01, p=0.22$. For the prediction of anhedonia, conclusions also remained the same without controlling for a history of depression.

We also tested the opposite direction, namely whether higher levels of depressive symptoms at baseline would predict higher levels of dampening and lower levels of positive rumination at follow-up (Table 5). First, for dampening, in the initial step, dampening measured at baseline and history of depression were entered (given that the cross-sectional results revealed a dampening difference between never-depressed and formerly/currently depressed individuals). Next, baseline DASS-D was entered. A positive history of depression predicted more dampening.
DASS-D had a small, marginally significant positive association with dampening at follow-up. ${ }^{3}$ If MASQ-Anhedonia was entered in the second step, it did not add significantly to the explained variance of RPA-Dampening at follow-up. Second, for positive rumination, baseline DASS-D as well as baseline MASQ-Anhedonia did not significantly predict positive rumination measured at follow-up. ${ }^{4}$

\section{Discussion}

The aims of this study were (1) to examine the concurrent relations between response styles to positive affect and depression (in terms of symptoms and history of depression) and (2) to explore the (bi-directionality of the) prospective relation between response styles to positive affect and depressive symptoms.

Let us first turn to the cross-sectional relations. Higher levels of dampening were related to higher levels of depressive symptoms, extending prior research mainly conducted in student samples (Feldman et al. 2008; Johnson et al. 2008; Raes et al. 2009; Werner-Seidler et al. 2013) and children (Bijttebier et al. 2012) to a large community sample. The size of the correlation resembles the correlations around 0.45 across these studies despite different populations and different measures of depressive symptoms. We found no evidence for a unique relation between dampening and anhedonic symptoms. Thus, at present, it is not definite that dampening has a privileged relation with the anhedonic part of depression. It is plausible that dampening not only influences symptoms related to positive affect but also increases negative affect or general distress. Relatedly, verbally analysing positive scenarios can increase feelings of anxiety (Holmes et al. 2009). However, we should note that we had

\footnotetext{
${ }^{3}$ For one participant, the standardised DFBeta value had an absolute value larger than 1 for the predictor DASS-D. Excluding this influential case revealed a significant effect of DASS-D on RPADampening measured at follow-up, $\beta=0.13, p=0.002$.

${ }^{4}$ To investigate the predictive value of dampening in a vulnerable sample, all prospective analyses were repeated with only the formerly depressed group $(n=75)$. If follow-up depressive symptoms were regressed on baseline depressive symptoms and the three scales of the RPA, then, again, only baseline depressive symptoms was a significant predictor. When predicting anhedonic symptoms, again, self-focused positive rumination had a significant negative association with depressive symptoms at follow-up $(\beta=-0.41, p=0.004)$. Unexpectedly, higher levels of Emotion-Focused positive rumination predicted more depressive symptoms $(\beta=0.31, p=0.03)$. Dampening remained a non-significant predictor $(\beta=-0.15, p=0.18)$. For the opposite pattern, depressive symptoms did not significantly predict dampening after controlling for dampening measured at baseline $(p=0.36)$, and similarly, anhedonic symptoms did not significantly predict dampening after controlling for dampening measured at baseline $(p=0.61)$.
} 
Table 5 Hierarchical regression analysis for the prediction of responses to positive affect at follow-up $n=293 . \mathrm{DV}=$ Dependent

variable. Dependent variables are measured at follow-up (T2). All predictors are measured at baseline (T1). Significant predictors are in bold. $\beta$ 's indicate the regression coefficients when predictors (except Past MDE) and the dependent variable are standardised. DASS-

$\mathrm{D}=$ Depression subscale of the Depression Anxiety Stress Scales, RPA $=$ Responses to Positive Affect Scale:

Dampening subscale of RPA

(D), Emotion-focused positive rumination subscale (EF), Selffocused positive rumination subscale of the RPA (SF);

MASQ-A = Anhedonic

Depression subscale of the

Short form of the Dutch adaptation of the Mood and Anxiety Symptoms Questionnaire (MASQ-D30).

Past MDE = history of depression: previous/current episode(s) or no previous episode

\begin{tabular}{|c|c|c|c|c|c|}
\hline & $B$ & SE $B$ & $\beta$ & $p$ & $R^{2}$ \\
\hline \multicolumn{6}{|c|}{$D V: R P A-D a m p e n i n g-T 2$} \\
\hline \multicolumn{6}{|l|}{ Step 1} \\
\hline RPA-D & 0.77 & 0.04 & 0.72 & $<0.001$ & \\
\hline Past MDE & 0.72 & 0.35 & 0.18 & 0.04 & 0.55 \\
\hline \multicolumn{6}{|l|}{ Step 2} \\
\hline RPA-D & 0.73 & 0.05 & 0.68 & $<0.001$ & \\
\hline Past MDE & 0.61 & 0.36 & 0.15 & 0.09 & \\
\hline DASS-D-T1 & 0.09 & 0.05 & 0.08 & 0.06 & 0.55 \\
\hline \multicolumn{6}{|l|}{ Step 2} \\
\hline RPA-D & 0.76 & 0.05 & 0.71 & $<0.001$ & \\
\hline Past MDE & 0.70 & 0.36 & 0.17 & 0.051 & \\
\hline MASQ-A & 0.01 & 0.02 & 0.01 & 0.77 & 0.55 \\
\hline \multicolumn{6}{|c|}{$D V:$ RPA-SelfFocus-T2 } \\
\hline \multicolumn{6}{|l|}{ Step 1} \\
\hline RPA-SF & 0.63 & 0.05 & 0.63 & $<0.001$ & \\
\hline Past MDE & -0.39 & 0.27 & -0.14 & 0.15 & 0.41 \\
\hline \multicolumn{6}{|l|}{ Step 2} \\
\hline RPA-SF & 0.62 & 0.05 & 0.63 & $<0.001$ & \\
\hline Past MDE & -0.37 & 0.27 & -0.13 & 0.18 & \\
\hline DASS-D-T1 & -0.01 & 0.04 & -0.01 & 0.76 & 0.41 \\
\hline \multicolumn{6}{|l|}{ Step 2} \\
\hline RPA-SF & 0.58 & 0.05 & 0.59 & $<0.001$ & \\
\hline Past MDE & -0.27 & 0.27 & -0.10 & 0.32 & \\
\hline MASQ-A & -0.03 & 0.02 & -0.10 & 0.06 & 0.42 \\
\hline \multicolumn{6}{|c|}{ DV: RPA-EmotionFocus-T2 } \\
\hline \multicolumn{6}{|l|}{ Step 1} \\
\hline RPA-EF & 0.60 & 0.04 & 0.64 & $<0.001$ & \\
\hline Past MDE & -0.05 & 0.26 & -0.02 & 0.86 & 0.41 \\
\hline \multicolumn{6}{|l|}{ Step 2} \\
\hline RPA-EF & 0.61 & 0.04 & 0.64 & $<0.001$ & \\
\hline Past MDE & -0.08 & 0.26 & -0.03 & 0.75 & \\
\hline DASS-D-T1 & 0.02 & 0.03 & 0.03 & 0.56 & 0.41 \\
\hline \multicolumn{6}{|l|}{ Step 2} \\
\hline RPA-EF & 0.57 & 0.05 & 0.60 & $<0.001$ & \\
\hline Past MDE & 0.05 & 0.26 & 0.02 & 0.85 & \\
\hline MASQ-A & -0.03 & 0.02 & -0.08 & 0.12 & 0.41 \\
\hline
\end{tabular}

The findings suggest that the anhedonic symptom part of depression relates negatively to both emotion-focused and self-focused positive rumination. We used the RPA as a measure of affect regulation, and the MASQ-A as a measure of anhedonic symptoms. Although from a different context (i.e., the MASQ-A does not assess what people think or do when feeling happy), some items that measure positive rumination (RPA) share content with the positively formulated MASQ-A. Correlations confirm that the items are not interchangeable, but we should monitor that questionnaires sufficiently differentiate between the of depression. 
different concepts (i.e., positive affect regulation and anhedonia).

Interestingly, not only the currently depressed group, but also the formerly depressed group had a greater tendency to dampen positive affect than people who had never experienced a depressive episode. In our sample, this latter difference was not due to differences in current levels of depressive symptoms (i.e., the two groups did not differ in their level of depressive symptoms and the dampening difference remained significant after controlling for depressive symptoms). As such, this may point to a difference in dampening due to having had a depressive episode or not (i.e., dampening as a scar). In this case, there should initially be no difference in terms of levels of dampening between individuals who will or will not develop a first depressive episode at a later point in time (Burcusa and Iacono 2007). However, another possibility is that there was already an initial difference (Burcusa and Iacono 2007). To conclude on this point, a very large sample of never-depressed individuals should be screened and assessed on a longitudinal basis. Notably, and as also reported by Werner-Seidler et al. (2013), the effect size for the difference between the formerly and never-depressed group was rather small $(d=0.40)$.

Considering the three depression groups together, people who had recovered dampened significantly more than a never-depressed group (see above), and also significantly less compared to a currently depressed group. Importantly, this latter difference was driven by the current depressive symptoms. This suggests that once people have had a depression, it is probably not the current diagnostic status that is most important, rather, it is the current level of depressive symptoms that relates to dampening.

With regard to positive rumination, consistent with previous research (Werner-Seidler et al. 2013), depression groups did not differ on their level of positive rumination (after controlling for depressive symptomatology). This implies that although people with a (past) depression are more likely to dampen positive affect than never-depressed controls, they may be able to engage in up-regulating strategies for positive affect to a similar extent as neverdepressed controls.

The results imply that therapy cannot solely focus on pleasant activities to enhance positive affect (because dampening might undo or counterwork such an intervention). Clinical interventions should additionally focus on how people reflect on this positive affect (see also for example in Dunn 2012; Feldman et al. 2008; Werner-Seidler et al. 2013). This would also be interesting in the context of relapse prevention given that dampening was characteristic of formerly depressed individuals and given that depression is a highly recurrent disorder (e.g., Burcusa and Iacono 2007).
Hitherto, the relation between responses to positive affect and depression has been studied mostly in cross-sectional studies. As such, more prospective studies are sorely needed to examine whether responses to positive affect also influence the course of depressive symptoms. This was another main goal of our study. Against predictions, however, the tendency to dampen positive affect was not predictive for future levels of depressive symptoms at 5 months. With regard to other types of responding to positive affect, a focus on positive aspects of the self and a focus on the positive feelings were also not predictive for later depressive symptoms. The latter is in accord with a study in remitted depressed patients (Gilbert et al. 2013) and two studies in students (Raes et al. 2012). The dampening results are in line with the finding that dampening did not predict depressive symptoms over a 3-month interval in children (Bijttebier et al. 2012). However, the dampening results are in conflict with the positive association found in two student samples with the same measures (Raes et al. 2012, study 1) and the same follow-up period (Raes et al. 2012, study 2). First, given that previous prospective studies did not control for past depression, it is possible that dampening has no predictive value above baseline depressive symptoms and history of depression. However, we also found no strong evidence for a relation between dampening and prospective levels of depressive symptoms when not controlling for a history of depression. Second, it is possible that the level of dampening interacts with the occurrence of significant positive events in the prediction of depressive symptoms (Raes et al. 2012), in analogy to vulnerability (e.g., depressive rumination) by stress interactions. This would imply that although positive events overall have a beneficial outcome in terms of lower levels of depressive symptoms, this effect is attenuated in people with a dampening cognitive style. Or, alternatively, experiencing fewer positive events could be buffered by an adaptive regulation style (confer Vines and Nixon 2009). Therefore, we would suggest for future research to include a measure of positive events. It is also important to elaborate here on a recent study of dampening in the context of a specific life event, i.e., giving birth (Raes et al. 2014). Prepartum dampening (not positive rumination) predicted depressive symptoms at 12 and 24 weeks postpartum, even when controlling for depression history and depressive symptoms prepartum (Raes et al. 2014). The regulation of positive affect might be especially predictive for postpartum depression, given that having a child is accompanied by both negative and positive emotions. Further, in the context of dampening thoughts in response to positive affect, it would be interesting to also collect information on the depression subtype. For example, when people suffer from an atypical depression, decreased reactivity to positive events is, by definition, not a central feature. Taken 
together, there is a clear need for more research on the conditions (i.e., moderating variables) that make dampening a detrimental factor in the course of depression, given that some studies do and others do not observe a prospective link between dampening and depression. Concerning the opposite direction, we also found no strong evidence that baseline levels of depressive symptoms predicted responses to positive affect measured 5 months later.

Although responses to positive affect did not predict depressive symptoms as measured by the DASS, lower levels of self-focused positive rumination predicted increased anhedonia levels (above baseline anhedonia and history of depression). This suggests that in the prevention of anhedonic symptoms, an exclusive focus on dampening in the context of positive affect regulation might be too strict. It was not so much how people tended to downward positive affect, but rather how they tended (not) to focus on positive aspects of the self in response to positive affect (i.e., "I am getting everything done") that appeared to be important.

We want to consider the questionnaires. The RPA, which was used to assess responses to positive affect, focuses on when one feels happy, excited, or enthused. It is unclear whether the current results also relate to other combinations of emotions, for example more attachmentrelated emotions such as love, tenderness, and gratitude. Next, the RPA assesses thoughts in response to positive affect. The scale does not make a distinction between responses to consummatory pleasure (i.e., the 'in-the-moment' pleasure in response to a positive stimulus) and to anticipatory pleasure (i.e., pleasure in anticipation of a positive stimulus). Given that these are supposed to be related but distinct facets of experiencing pleasure (e.g., Bryant 2003; Gard et al. 2006), future investigation could reveal (1) to what extent thoughts in response to these different types of pleasure or positive feelings are interrelated and (2) whether (dampening) thoughts in response to consummatory versus anticipatory pleasure relate differently to symptom clusters of depression. Also, using the RPA, participants have to judge how they generally respond to positive affect. A more ecological valid measure would be to use the Experience Sampling Method (ESM), measuring thoughts people have in response to daily life positive events (i.e., dampening as well as self-focused or emotion-focused responses). This would add to the preliminary prospective evidence that the level of positive affect following positive life events (measured via the ESM) predicts depression and anxiety symptoms (Wichers et al. 2010).

The MASQ-D30, used to assess anhedonia in our study, was developed in line with the tripartite model of Clark and Watson (1991) and its anhedonic sale aims to measure (a lack of) positive affect. These anhedonic items differ from scales from other traditions such as the Snaith-Hamilton Pleasure Scale (SHAPS, Franken et al. 2007; Snaith et al. 1995). The SHAPS focuses on the level of pleasure or reward derived from various experiences (e.g., watching one's favourite television programme). In the present study, dampening thoughts did not predict later anhedonic symptoms as measured by the MASQ. As such, we suggest to investigate in future research whether dampening thoughts relate to other dimensions of anhedonia as measured, for example, by the SHAPS.

Several limitations warrant mention. A first limitation of the present study may be that a part of the participants was recruited via the network of the researcher. Although it was mentioned in the informed consent that analyses will be conducted anonymously, we cannot exclude that some participants might have answered differently due to being acquainted with the researcher. Another sampling limitation is that participants were not systematically selected. Our study is also limited by the reliance on self-report questionnaires. Most importantly, this means that participants were assigned to a group of current, past or no depression based on a self-report questionnaire and not based on a diagnostic interview administered by an experienced clinician (e.g., the SCID; First et al. 1996). While the latter would be preferred, the current method allowed us to reach a larger sample. We acknowledge that the MDQ cannot be regarded as equal to a full diagnostic assessment by a clinician. Therefore, it cannot be ensured that the same pattern of results would appear when a non-self-report measure to assess depressive status was used. Also, the study was conducted via internet which means that participants were unmonitored. Next, we did not administer information on other forms of psychopathology than depression and, therefore, we cannot control for comorbidity. For example, dysregulation of positive affect also plays a role in other disorders such as bipolar disorder (e.g., Carl et al. 2013; Edge et al. 2013; Gilbert et al. 2013; Gruber et al. 2011) and it is likely that the responses styles can not be categorised as adaptive or maladaptive in general. Finally, our sample was highly educated, limiting generalisation of the results.

To conclude, the current study confirms the robust finding that dampening responses to positive affect are positively related to depressive symptoms. Importantly, we found that not only currently depressed, but also recovered depressed individuals continue to report higher levels of dampening responses as compared to never-depressed controls. Future research will need to examine whether this reflects a scar phenomenon in the recovered group or points to a risk or vulnerability factor that was already initially present in that group. Positive rumination did not differ depending on depressive status. Furthermore, we found no 
evidence that dampening predicts depressive symptoms after 5 months, suggesting that more research is needed to clarify the precise role of dampening in the course of depression. Positive rumination, however, predicted later anhedonic symptoms, such that lower levels of self-focused positive rumination predicted higher future anhedonic symptoms.

Acknowledgments This work was supported by a research funding of the Research Foundation-Flanders (FWO; PhD fellowship 2010-2014 to Sabine Nelis and Grant G.0923.12 to Patricia Bijttebier). Emily Holmes is supported by the Medical Research Council (United Kingdom) intramural program [MC-A060-5PR50], a Wellcome Trust Clinical Fellowship [WT088217], and the National Institute for Health Research (NIHR) Oxford Biomedical Research Centre Programme. The views expressed are those of the author(s) and not necessarily those of the NHS, the NIHR or the Department of Health.

Conflict of Interest Sabine Nelis, Emily A. Holmes, and Filip Raes declare that they have no conflict of interest.

Informed Consent All procedures followed were in accordance with the ethical standards of the responsible committee on human experimentation and informed consent was obtained from each participant.

Animal Rights No animal studies were carried out by the authors for this article.

\section{References}

Aldao, A., Nolen-Hoeksema, S., \& Schweizer, S. (2010). Emotionregulation strategies across psychopathology: A meta-analytic review. Clinical Psychology Review, 30, 217-237. doi:10.1016/j. cpr.2009.11.004

American Psychiatric Association. (1994). Diagnostic and statistical manual of mental disorders (4th ed.). Washington, DC: American Psychiatric Association.

American Psychiatric Association. (2013). Diagnostic and statistical manual of mental disorders (5th ed.). Washington, DC: American Psychiatric Association.

Bijttebier, P., Raes, F., Vasey, M. W., \& Feldman, G. C. (2012). Responses to positive affect predict mood symptoms in children under conditions of stress: A prospective study. Journal of Abnormal Child Psychology, 40, 381-389. doi:10.1007/s10802011-9579-2

Bryant, F. B. (2003). Savoring Beliefs Inventory (SBI): A scale for measuring beliefs about savouring. Journal of Mental Health, 12, 175-196. doi:10.1080/0963823031000103489

Burcusa, S. L., \& Iacono, W. C. (2007). Risk for recurrence in depression. Clinical Psychology Review, 27, 959-985. doi:10. 1016/j.cpr.2007.02.005

Carl, J. R., Soskin, D. P., Kerns, C., \& Barlow, D. H. (2013). Positive emotion regulation in emotional disorders: A theoretical review. Clinical Psychology Review, 33, 343-360. doi:10.1016/j.cpr. 2013.01.003

Clark, L. A., \& Watson, D. (1991). Tripartite model of anxiety and depression: Psychometric evidence and taxonomic implications. Journal of Abnormal Psychology, 100, 316-336. doi:10.1037// 0021-843X.100.3.316 de Beurs, E., van Dyck, R., Marquenie, L. A., Lange, A., \& Blonk, R. (2001). De DASS: Een vragenlijst voor het meten van depressie, angst en stress [The DASS: A questionnaire for the measurement of depression, anxiety and stress]. Gedragstherapie, 34, 35-53.

Dunn, B. D. (2012). Helping depressed clients reconnect to positive emotion experience: Current insights and future directions. Clinical Psychology \& Psychotherapy, 19, 326-340. doi:10. 1002/cpp.1799

Eberhard-Gran, M., Eskild, A., Tambs, K., Samuelsen, S. O., \& Opjordsmoen, S. (2002). Depression in postpartum and nonpostpartum women: Prevalence and risk factors. Acta Psychiatrica Scandinavica, 106, 426-433. doi:10.1034/j.1600-0447. 2002.02408.x

Edge, M. D., Miller, C. J., Muhtadie, L., Johnson, S. L., Carver, C. S., Marquinez, N., \& Gotlib, I. H. (2013). People with bipolar I disorder report avoiding rewarding activities and dampening positive emotion. Journal of Affective Disorders, 146, 407-413. doi:10.1016/j.jad.2012.07.027

Eisner, L. R., Johnson, S. L., \& Carver, C. S. (2009). Positive affect regulation in anxiety disorders. Journal of Anxiety Disorders, 23, 645-649. doi:10.1016/j.janxdis.2009.02.001

Feldman, G. C., Joormann, J., \& Johnson, S. L. (2008). Responses to positive affect: A self-report measure of rumination and dampening. Cognitive Therapy and Research, 32, 507-525. doi:10.1007/s10608-006-9083-0

First, M. B., Spitzer, R. L., Gibbon, M., \& Williams, J. B. W. (1996). Structured clinical interview for DSM-IV axis I disorders. Washington, DC: American Psychiatric Association.

Franken, I. H. A., Rassin, E., \& Muris, P. (2007). The assessment of anhedonia in clinical and non-clinical populations: Further validation of the Snaith-Hamilton Pleasure Scale (SHAPS). Journal of Affective Disorders, 99, 83-89. doi:10.1016/j.jad. 2006.08.020

Gard, D. E., Germans Gard, M., Kring, A. M., \& John, O. P. (2006). Anticipatory and consummatory components of the experience of pleasure: A scale development study. Journal of Research in Personality, 40, 1086-1102. doi:10.1016/j.jrp.2005.11.001

Gentzler, A. L., Morey, J. N., Palmer, C. A., \& Yi, C. Y. (2012). Young adolescents' responses to positive events: Associations with positive affect and adjustment. The Journal of Early Adolescence, 33, 663-683. doi:10.1177/0272431612462629

Gilbert, K. E. (2012). The neglected role of positive emotion in adolescent psychopathology. Clinical Psychology Review, 32, 467-481. doi:10.1016/j.cpr.2012.05.005

Gilbert, K. E., Nolen-Hoeksema, S., \& Gruber, J. (2013). Positive emotion dysregulation across mood disorders: How amplifying versus dampening predicts emotional reactivity and illness course. Behaviour Research and Therapy, 51, 736-741. doi:10. 1016/j.brat.2013.08.004

Gruber, J., Eidelman, P., Johnson, S. L., Smith, B., \& Harvey, A. G. (2011). Hooked on a feeling: Rumination about positive and negative emotion in inter-episode bipolar disorder. Journal of Abnormal Psychology, 120, 956-961. doi:10.1037/a0023667

Hechtman, L. A., Raila, H., Chiao, J., \& Gruber, J. (2013). Positive emotion regulation and psychopathology: A transdiagnostic cultural neuroscience approach. Journal of Experimental Psychopathology, 4, 1-27. doi:10.5127/jep.030412

Holmes, E. A., Lang, T. J., \& Shah, D. M. (2009). Developing interpretation bias modification as a "cognitive vaccine" for depressed mood: Imagining positive events makes you feel better than thinking about them verbally. Journal of Abnormal Psychology, 118, 76-88. doi:10.1037/a0012590

Johnson, S. L., McKenzie, G., \& McMurrich, S. (2008). Ruminative responses to negative and positive affect among students diagnosed with bipolar disorder and major depressive disorder. 
Cognitive Therapy and Research, 32, 702-713. doi:10.1007/ s10608-007-9158-6

Karsten, J., Hartman, C. A., Smit, J. H., Zitman, F. G., Beekman, A. T. F., Cuijpers, P., et al. (2011). Psychiatric history and subthreshold symptoms as predictors of the occurrence of depressive or anxiety disorder within 2 years. The British Journal of Psychiatry, 198, 206-212. doi:10.1192/bjp.bp.110. 080572

Lovibond, S. H., \& Lovibond, P. F. (1995). Manual for the Depression Anxiety Stress Scales (2nd ed.). Sydney: Psychology Foundation.

Nelis, S., Debeer, E., Holmes, E. A., \& Raes, F. (2013). Dysphoric students show higher use of the observer perspective in their retrieval of positive versus negative autobiographical memories. Memory, 21, 423-430. doi:10.1080/09658211.2012.730530

Nieuwenhuijsen, K., de Boer, A. G. E. M., Verbeek, J. H. A. M., Blonk, R. W. B., \& van Dijk, F. J. H. (2003). The Depression Anxiety Stress Scales (DASS): Detecting anxiety disorder and depression in employees absent from work because of mental health problems. Occupational and Environmental Medicine, 60, 77-82. doi:10.1136/oem.60.suppl_1.i77

Nolen-Hoeksema, S. (1991). Responses to depression and their effects on the duration of depressive episodes. Journal of Abnormal Psychology, 100, 569-582. doi:10.1037/0021-843X.100.4.569

Nolen-Hoeksema, S., Wisco, B. E., \& Lyubomirsky, S. (2008). Rethinking rumination. Perspectives on Psychological Science, 3, 400-424. doi:10.1111/j.1745-6924.2008.00088.x

Raes, F., Daems, K., Feldman, G. C., Johnson, S. L., \& Van Gucht, D. (2009). A psychometric evaluation of the Dutch version of the responses to positive affect questionnaire. Psychologica Belgica, 49, 293-310. doi:10.5334/pb-49-4-293

Raes, F., Smets, J., Nelis, S., \& Schoofs, H. (2012). Dampening of positive affect prospectively predicts depressive symptoms in non-clinical samples. Cognition and Emotion, 26, 75-82. doi:10. 1080/02699931.2011.555474

Raes, F., Smets, J., Wessel, I., Van Den Eede, F., Nelis, S., Franck, E., et al. (2014). Turning the pink cloud grey: Dampening of positive affect predicts postpartum depressive symptoms. Journal of Psychosomatic Research, 77, 64-69. doi:10.1016/j. jpsychores.2014.04.003

Snaith, R. P., Hamilton, M., Morley, S., Humayan, A., Hargreaves, D., \& Trigwell, P. (1995). A scale for the assessment of hedonic tone the Snaith-Hamilton Pleasure Scale. The British Journal of Psychiatry, 167, 99-103. doi:10.1192/bjp.167.1.99

Thomsen, K. D. (2006). The association between rumination and negative affect: A review. Cognition and Emotion, 20, 1216-1235. doi:10.1080/02699930500473533

Van der Does, A. J. W., Barnhofer, T., \& Williams, J. M. G. (2003). The Major Depression Questionnaire (MDQ). Retrieved from http://www.dousa.nl/publications.htm

Vines, L., \& Nixon, R. D. V. (2009). Positive attributional style, life events and their effect on children's mood: Prospective study. Australian Journal of Psychology, 61, 211-219. doi:10.1080/ 00049530802579507

Wardenaar, K. J., Veen, T. V., Giltay, E. J., Beurs, E. D., Penninx, B. W. J. H., \& Zitman, F. G. (2010). Development and validation of a 30-item short adaptation of the Mood and Anxiety Symptoms Questionnaire (MASQ). Psychiatry Research, 179, 101-106. doi:10.1016/j.psychres.2009.03.005

Watson, D., Clark, L. A., Weber, K., Assenheimer, J. S., Strauss, M. E., \& McCormick, R. A. (1995a). Testing a tripartite model: II. Exploring the symptom structure of anxiety and depression in student, adult, and patient samples. Journal of Abnormal Psychology, 104, 15-25. doi:10.1037/0021-843X.104.1.15

Watson, D., Weber, K., Assenheimer, J. S., Clark, L. A., Strauss, M. E., \& McCormick, R. A. (1995b). Testing a tripartite model: I. Evaluating the convergent and discriminant validity of anxiety and depression symptom scales. Journal of Abnormal Psychology, 104, 3-14. doi:10.1037/0021-843X.104.1.3

Werner-Seidler, A., Banks, R., Dunn, B. D., \& Moulds, M. L. (2013). An investigation of the relationship between positive affect regulation and depression. Behaviour Research and Therapy, 51, 46-56. doi:10.1016/j.brat.2012.11.001

Wichers, M., Peeters, F., Geschwind, N., Jacobs, N., Simons, C. J. P., Derom, C., et al. (2010). Unveiling patterns of affective responses in daily life may improve outcome prediction in depression: A momentary assessment study. Journal of Affective Disorders, 124, 191-195. doi:10.1016/j.jad.2009.11.010

Williams, J. M. G., Van der Does, A. J. W., Barnhofer, T., Crane, C., \& Segal, Z. S. (2008). Cognitive reactivity, suicidal ideation and future fluency: Preliminary investigation of a differential activation theory of hopelessness/suicidality. Cognitive Therapy and Research, 32, 83-104. doi:10.1007/s10608-006-9105-y 